This is a post-peer-review, pre-copyedit version of an article published in Applied Research in Quality of Life. The final authenticated version is available online at: https://doi.org/10.1007/s11482-020-09845-y. This publication is embargoed until 29/5/2021.

Nevarez-Flores, A.G., Morgan, V.A., Harvey, C. et al. Health-Related Quality of Life, Functioning and Social Experiences in People with Psychotic Disorders. Applied Research Quality Life (2020). https://doi.org/10.1007/s11482-020-09845-y 


\section{Health-Related Quality of Life, Functioning and Social Experiences in People with Psychotic Disorders}

Adriana G. Nevarez-Flores MD', Vera A. Morgan $\mathrm{PhD}^{2,3,1}$, Carol Harvey $\mathrm{MD}^{4,5}$, Monique Breslin $\mathrm{PhD}^{1}$, Vaughan J.

Carr $\mathrm{MD}^{6,7}$, Kristy Sanderson $\mathrm{PhD}^{8,1}$, Anna Waterreus GradDipClinEpi², Amanda L. Neil PhD ${ }^{*, 1}$

${ }^{1}$ Menzies Institute for Medical Research, University of Tasmania, Hobart, Tasmania, Australia

${ }^{2}$ Neuropsychiatric Epidemiology Research Unit, School of Population and Global Health, The University of Western

Australia, Crawley, Western Australia, Australia

${ }^{3}$ Centre for Clinical Research in Neuropsychiatry, Division of Psychiatry, Medical School, The University of Western

Australia, Crawley, Western Australia, Australia

${ }^{4}$ Psychosocial Research Centre, Department of Psychiatry, The University of Melbourne, Melbourne, Victoria, Australia

${ }^{5}$ North Western Mental Health, Melbourne, Victoria, Australia

${ }^{6}$ Research Unit for Schizophrenia Epidemiology, School of Psychiatry, University of New South Wales, St Vincent's

Hospital, Darlinghurst Sydney, New South Wales, Australia

${ }^{7}$ Department of Psychiatry, School of Clinical Sciences, Monash Medical Centre, Monash University, Clayton Melbourne, Victoria, Australia

${ }^{8}$ School of Health Sciences, University of East Anglia. Norwich Research Park Norwich, NR4 7TJ, UK

*Corresponding author e-mail address: Amanda.Neil@utas.edu.au

\section{ORCID:}

A. G. Nevarez-Flores: 0000-0003-2217-1401

V.A. Morgan: 0000-0003-3600-9042

C. Harvey: 0000-0002-5066-8450

M. Breslin: 0000-0002-8135-3136

V. J. Carr: 0000-0002-8907-5804

K. Sanderson: 0000-0002-3132-2745

A. Neil: 0000-0002-1344-6672 


\section{Acknowledgments}

The Survey of High Impact Psychosis was funded by the Australian Government Department of Health and Ageing. The study sponsor had a role in the design of the original survey but did not have any role in the collection of the study data, or the design or interpretation of the data analysis reported here, or in the writing of this paper or the decision to submit this paper for publication. This publication is based on data collected in the framework of the 2010 Australian National Survey of High Impact Psychosis. The members of the Survey of High Impact Psychosis Study Group at the time were: V. Morgan (National Project Director), A. Jablensky (Chief Scientific Advisor), A. Waterreus (National Project Coordinator), R. Bush, V. Carr, D. Castle, M. Cohen, C. Galletly, C. Harvey, B. Hocking, A. Mackinnon, P. McGorry, J. McGrath, A. Neil, S. Saw, H. Stain. Ethics approvals for the study were obtained from relevant institutional human research ethics committees. The study was funded by the Australian Government Department of Health and Ageing. This report acknowledges, with thanks, the hundreds of mental health professionals who participated in the preparation and conduct of the survey and the many Australians with psychotic disorders who gave their time and whose responses form the basis of this publication.

Conflict of Interest: The authors declare that they have no conflict of interest. 


\begin{abstract}
To inform development of a model for health-related quality of life (HRQoL) in people with psychotic disorders, we aimed to assess correlations between utilities and dimension scores for the Assessment of Quality of Life (AQoL)-4D with functioning and social experiences; ascertain if patient housing and clinical status affected correlations; and determine aspects of functioning that jointly predict HRQoL. We analyzed data for 1,642 people with an ICD-10 psychotic disorder from the 2010 Australian National Survey of Psychosis. Global functioning was measured with the Personal and Social Performance scale, independent functioning with the Multidimensional Scale of Independent Functioning and social functioning through level of social dysfunction. Social experiences comprised perceived loneliness and experienced stigma. We assessed Spearman's rank correlation coefficients and undertook linear regression analyses. Moderate associations were found between AQoL-4D utilities and all variables, except experienced stigma. Perceived loneliness had the strongest association. The AQoL-4D social relationships dimension was most strongly associated with social variables; its independent living dimension with global and independent functioning. Correlations between utilities and all variables, except for social dysfunction, were modified by housing. Course of disorder impacted correlations with utilities and independent functioning. Global functioning and social dysfunction were found to jointly predict HRQoL. In conclusion, as the AQoL-4D can differentiate between functioning and social experiences individually and when categorized by housing and clinical status in people with psychosis, predictive models of HRQoL in this population are feasible, and only need include select aspects of functioning and social experiences, particularly perception of loneliness.
\end{abstract}

Keywords: Psychotic disorders, quality of life, functioning, loneliness, AQoL-4D, multi-attribute utility instrument. 


\section{Introduction}

Psychotic disorders are some of the most debilitating and chronic mental disorders. Impairment in global functioning, i.e., an individual's ability to perform their roles across several domains/types of functioning (Aas 2010), has been recognized as a characteristic of all mental disorders (World Health Organization 1992), and as a specific criterion for diagnosis in psychotic disorders (American Psychiatric Association 2013; Petho and Ban 1988). Global functioning encompasses occupational, social and independent functioning as well as behaviour and adaptive functioning. Independent functioning encompasses role responsibility, support, and performance within work, education, and residential domains (Jaeger et al. 2003) and is thus directly related to, but not as broad, as global functioning. Quality of life (QoL), “an individual's perception of their position in life in the context of the culture and value systems in which they live and in relation to their goals, expectations, standards and concerns" (World Health Organization 1997), is also affected in people living with a psychotic disorder. Given that "psychological and social aspects, functional capacity and symptomatology all relate to QoL" (Fayers and Machin 2016), a discernible relationship between each of these elements with QoL is expected.

In a recent systematic review of people with psychotic disorders, a relationship between global functioning and QoL has been confirmed (Nevarez-Flores et al. 2019), with the strength of the association (ranging from small to strong) dependent upon the QoL instrument employed. There was also evidence that the strength of the association may vary by the individual's housing and clinical status, and that the social domains of QoL are most strongly associated with global functioning. Global functioning was also assessed as a predictor of overall QoL in nine articles, and identified as a predictor in seven.

Health-related quality of life (HRQoL), focusses on the effects of disease or treatment on QoL (Fayers and Machin 2016). HRQoL has elsewhere been identified as having the strongest relationship with independent functioning, across 20 subjective and objective general and illness related characteristics, in people with a psychotic disorder based on effect size (Neil et al. 2018). Within this study, a large effect was also found for social functioning, however, global functioning was not considered. Meanwhile, relationships with symptomatology were clearly established, with reduction in HRQoL associated with increasing numbers of negative symptoms and cumulative effects observed for the co-occurrence of mania and positive and/or depressive symptoms. Within this study, HRQoL was assessed with the Assessment of Quality of Life (AQoL)-4D (Hawthorne et al. 1999), a multi-attribute utility instrument (i.e., a preference-based measure of health status) validated in people with psychotic disorders (Herrman et 
al. 2002). Neil et al. (2018) concluded that the AQoL-4D was sensitive and superior to other utility instruments used in people with psychosis. The AQoL-4D may thus enable the construction of valid and reliable predictive models of HRQoL and the first for health state utilities, in people with psychotic disorders.

Social experiences are also important for the QoL/HRQoL of people with psychotic disorders (Nevarez-Flores et al. 2019), with feelings of loneliness more common in this population than the general population (Chrostek et al. 2016). Further, self-stigma of people with schizophrenia has been found to affect their QoL (Lin et al. 2016). However, the relationship between perception of loneliness and experienced stigma with the HRQoL of people with psychotic disorders has not been fully elucidated. Indeed, loneliness has elsewhere been identified as unable to be captured in the assessment of quality-adjusted life years (QALYs) (McDaid et al. 2017), which are calculated using health state utilities.

Extending on previous work, this study is therefore intended to establish the capacity of the AQoL-4D to be employed in predictive models of HRQoL in people with psychosis, and act as a preliminary stage towards development of a comprehensive theoretical model of the causal pathway for HRQoL in this population. Using data captured in the 2010 Australian National Survey of Psychosis, we therefore aim to: 1) further explore the relationship between different types of functioning and social experiences (i.e. perceived loneliness and experienced stigma) with HRQoL assessed with the AQoL-4D by assessing correlations between these variables; 2) determine if housing and clinical status (assessed through mental health services used in the last 12-months and course of disorder) affect this relationship and, 3) ascertain the types of functioning that jointly predict the HRQoL of this population to aid parsimonious model development. We hypothesized there would be a moderate relationship between HRQoL (AQoL4D utilities) and different aspects of functioning, and that the social dimension of the AQoL-4D would be the most strongly related with all aspects of functioning. We further hypothesized that housing and clinical status would impact on correlations.

\section{Methods}

\section{Study design and participants}

This study analyzed data collected as part of the 2010 Australian National Survey of Psychosis (Morgan et al. 2012; Morgan et al. 2014), which was undertaken at seven sites in five Australian states, and covered an estimated resident population of $1,464,923$ people — about $10 \%$ of the Australian population aged $18-64$ years. A two-phase design was 
employed. In Phase 1 (March 2010), public specialized mental health services - emergency, inpatient, outpatient, ambulatory and community mental health services (CMHS) and non-government organizations supporting people with mental illness - were screened for individuals likely to meet diagnostic criteria for psychosis. Administrative records were also screened to identify individuals with psychosis who were in contact with public mental health services in the 11-months before census but not in the census month. In Phase 2, people who screened positive for psychosis in Phase 1 were randomly selected for interview.

A total of 7,955 individuals were screen-positive for psychotic illness in Phase 1 . Of these, 1,825 were randomly selected for interview by age group and site, and gave written informed consent after receiving full information on the study; 1,642 met ICD-10 criteria for a psychotic disorder. The interview was developed based on reliable, available, and cost and time effective measures and administered by mental health professionals and comprised more than 1,500 items. Data items covered symptomatology, substance use, functioning, disability, cognitive ability, mental and physical health, service utilization, medication use, education, employment, housing, and HRQoL. The survey variables (items) selected for inclusion in this study were chosen based on the available literature. The study was approved by institutional human research ethics committees at all study sites. For full methodological details see Morgan and colleagues (2012; 2014).

\section{Variables}

\section{Health-Related Quality of Life}

HRQoL was assessed with the AQoL-4D, which gives rise to a preference-based measure of health status (utility) in the last 7-days, based on responses across four dimensions: independent living, psychological well-being, relationships and physical senses. Utilities can range from -0.04 for states worse than death through to 1.00 for full health. The AQoL-4D can provide profile scores for each dimension and has been validated for self- and interviewer administration (AQoL 2014). Interviewer administration was employed in this study.

\section{Functioning}

All specific assessments of functioning within the national survey were included in this study, comprising two functioning scales as well as participants' descriptions of their relationships with others and engagement in social activities. 
The Personal and Social Performance scale (PSP) as the broadest functioning scale employed in the survey, was used for the assessment of global functioning. The PSP is a 100-point single-item rating scale for measuring personal and social functioning within the last 12-months across four domains: socially useful activities including work and study, personal and social relationships, self-care, and disturbing and aggressive behaviours (Morosini et al. 2000). The scale is subdivided into 10 equal intervals ranging from 1, reflecting a lack of autonomy in basic functioning with extreme behaviours and survival risk, to 100 reflecting excellent functioning in all four domains.

The Multidimensional Scale of Independent Functioning (MSIF) was used for the assessment of independent functioning across work, education and residential domains in the last 4-weeks. Employing a semi-structured interview three dimensions are rated for each domain: quality of performance, assistance received and quality of the productive activities in each domain. Dimensions are rated along a seven-point scale in which 1 is normal functioning and 7 total disability. Individual global ratings from the three domains contribute to the overall global rating of functional disability (Jaeger et al. 2003). The MSIF thus does not comprehensively assess social functioning.

Social functioning was assessed based on participants' descriptions of their relationships with others and engagement in social activities, overall socializing, in the last 12-months. Participants were asked: how have they been getting on with other people; if they have been going out on any social activities; if they usually wait for others to ask him/her to do something with them or if they look for people to do things with; if they have met any friends and; how much time they spent alone. Based on the responses, interviewers then rated the participants' social dysfunction across four categories: no dysfunction, obvious dysfunction, severe dysfunction and uncertain.

\section{Social Experiences}

Perceived loneliness over the last 12-months was assessed through the question "In the last 12 months have you felt lonely? with the choice of five responses (plenty of friends, not lonely; friends, lonely occasionally; some friends but

lonely for company; socially isolated and lonely; not known). Experienced stigma in the last 12-months was assessed through the question "In the last 12 months have you experienced stigma or discrimination because of mental illness? with the choice of three responses (yes, no, not known).

\section{Housing}


Current housing referred to whether, at the time of interview, the individual resided in a public or private rented house/unit/apartment, family home, own house/unit, supported accommodation, institution or hospital, was homeless, or living in other housing (including living in a caravan/houseboat but not prison) (Harvey et al. 2012).

\section{Clinical status}

Two different proxy variables for clinical status were employed: 12-month service utilization and course of disorder. Service utilization was based on a 7-level hierarchy of mental health (MH) service use: MH inpatient admission, specialised MH emergency services (including crisis home visits), presentation to an emergency department (ED) with a MH problem, community MH services (CMHS), $\mathrm{MH}$ outpatient services, attendance at a general practitioner for $\mathrm{MH}$ issues (GPMH) and "Non-specified services", a category for participants not included in the previous groups. Course of disorder was rated as single episode/good recovery, multiple episodes/good recovery, multiple episodes/partial recovery, continuous illness/without deterioration or continuous illness/with deterioration.

\section{Statistical analysis}

Spearman's rank correlation coefficients were used to assess the strength of monotonic associations between AQoL4D utilities and dimension scores and different types of functioning and social experiences. Because of the skewed distribution of the data, Spearman's rank correlation coefficients were assessed. Correlations were also stratified by current housing, 12-month service utilization, and course of disorder. Strength of correlation was assessed according to the specifications of Cohen (Cohen 1988) established as $0.10 \leq r<0.3$ small effect, $0.3 \leq r<0.5$ medium effect, and $0.50 \leq r$ large effect, with significance set as $p<0.05$.

Using linear regression, global, independent and social functioning were tested for a univariable association with AQoL-4D utilities. Variables were required to achieve both significance at $p<0.05$ and the minimally important difference for the AQoL-4D (0.06) (Hawthorne and Osborne 2005; Neil et al. 2018) for further inclusion in a multivariable analysis. Missing values (less than 1\%), were excluded from the regression analyses. All analyses were conducted with Stata release 15.0.

\section{Results}

\section{Participant characteristics}


The HRQoL, functioning and social characteristics of the 1,642 participants with a psychotic disorder are described in Online Resource Table 1. The mean utility was 0.50, and among the AQoL-4D dimensions, social relationships had the lowest score. Significant functional impairment was observed with the PSP (Mean=56.22, SD=14.71), the MSIF (51\% at least moderate disability), and social dysfunction (64\% with some degree of dysfunction). The demographic and clinical characteristics of the sample are described in Online Resource Table 2.

\section{Correlations between AQoL-4D utilities and dimension scores with functioning, and social experiences}

Correlations between all variables are presented in Table 1. The largest correlation $(r=0.83)$ was between AQoL-4D utilities and social relationships dimension scores, followed by the correlation between the two functioning instruments $(r=-0.81)$. The smallest (negligible) correlations were between the functioning instruments and experienced stigma.

Correlations between AQoL-4D utilities and functioning and social experiences

All associations were moderate with one exception, experienced stigma, which was weak. The largest correlation, bordering on a large effect, was with perceived loneliness $(r=-0.49)$.

\section{Correlations between AQoL-4D dimension scores and functioning and social experiences}

The strength of the association with functioning and social experiences varied among dimensions of the AQoL-4D. The largest correlation coefficient $(r=-0.54)$ was between the social relationships dimension and perceived loneliness, followed by its correlation with social dysfunction. The smallest correlation $(r=-0.06)$ was between the psychological wellbeing dimension and the MSIF.

The functioning instruments were most strongly associated with the independent living dimension $(r=0.37$, $r=-0.33$, for the PSP and MSIF, respectively), followed by the social relationships dimension $(r=0.34, r=-0.26)$. The absolute magnitude of the coefficients was larger for the PSP than MSIF for all correlations. 
Table 1 Correlation coefficients between HRQoL measured with the AQoL-4D and global functioning measured with the PSP, independent functioning measured with the MSIF, social functioning measured through social dysfunction and social experiences in the last 12-months

\begin{tabular}{|c|c|c|c|c|c|c|c|c|c|c|}
\hline & \multicolumn{5}{|c|}{ AQoL } & \multirow{2}{*}{ PSP } & \multirow{2}{*}{ MSIF } & \multirow{2}{*}{ Soc } & \multirow{2}{*}{ Lonely } & \multirow{2}{*}{ Stigma } \\
\hline & IL & SR & PS & PW & Utilities & & & & & \\
\hline AQoL Independent Living (IL) & 1.00 & & & & & & & & & \\
\hline AQoL Social Relationships (SR) & $0.24 * * *$ & 1.00 & & & & & & & & \\
\hline AQoL Physical Senses (PS) & $0.28^{* * *}$ & $0.20^{* * *}$ & 1.00 & & & & & & & \\
\hline AQoL Psychological Wellbeing (PW) & $0.29 * * *$ & $0.31^{* * *}$ & $0.26^{* * *}$ & 1.00 & & & & & & \\
\hline AQoL Utilities & $0.53^{* * *}$ & $0.83^{* * *}$ & $0.39^{* * *}$ & $0.59^{* * *}$ & 1.00 & & & & & \\
\hline PSP Total range & $0.37^{* * *}$ & $0.34 * * *$ & $0.13^{* * *}$ & $0.09^{* * *}$ & $0.39^{* * *}$ & 1.00 & & & & \\
\hline $\begin{array}{l}\text { MSIF Overall global independent } \\
\text { functioning }\end{array}$ & $-0.33 * * *$ & $-0.26 * * *$ & $-0.13 * * *$ & $-0.06^{*}$ & $-0.32 * * *$ & $-0.81 * * *$ & 1.00 & & & \\
\hline $\begin{array}{l}\text { Social dysfunction in the last } 12- \\
\text { months (Soc) }\end{array}$ & $-0.15 * * *$ & $-0.42 * * *$ & $-0.14 * * *$ & $-0.12 * * *$ & $-0.39 * * *$ & $-0.34 * * *$ & $0.30 * * *$ & 1.00 & & \\
\hline $\begin{array}{l}\text { Perceived loneliness in the last } 12 \text { - } \\
\text { months (Lonely) }\end{array}$ & $-0.15 * * *$ & $-0.54 * * *$ & $-0.12 * * *$ & $-0.29 * * *$ & $-0.49 * * *$ & $-0.14 * * *$ & $0.09 * * *$ & $0.40^{* * *}$ & 1.00 & \\
\hline $\begin{array}{l}\text { Experienced stigma in the last } 12 \text { - } \\
\text { months }\end{array}$ & $-0.11 * * *$ & $-0.22 * * *$ & $-0.13 * * *$ & $-0.22 * * *$ & $-0.23 * * *$ & -0.03 & 0.01 & $0.08 * *$ & $0.21 * * *$ & 1.00 \\
\hline
\end{tabular}

AQoL, Assessment of Quality of Life; MSIF, Multidimensional Scale of Independent Functioning; PSP, Personal and Social Performance

Reported correlations are statistically significant at the ${ }^{*} p<0.05,{ }^{* *} p<0.01,{ }^{* * *} p<0.001$ 
Correlations between AQoL-4D utilities and functioning and social experiences stratified by possible effect modifiers

In general, correlations between AQoL-4D utilities with functioning and social experiences showed variation when assessed by current housing status, except for social dysfunction (Fig. 1). The greatest variation in correlations was observed for perceived loneliness, ranging from small $(r=-0.18)$ for the institution/hospital group to the strongest of all associations for current housing $(r=-0.69)$, for the other housing group. The smallest, negligible, correlation was observed for experienced stigma for the institution/hospital group ( $r=-0.01)$. Institutional care and the marginal housing category "other" gave rise to the extremes for experienced stigma and perceived loneliness. Correlations for family home, own house/unit and supported accommodation groups showed little variation across variables, being moderate ( $r=-0.32$ to -0.47$)$, except for experienced stigma, which were small. Correlations for public and private rented house/unit/apartment groups showed little variation between them. The greatest variability was for the other housing group ranging from negligible to large ( $r=-0.04$ to -0.69$)$, and the largest of all correlations. 


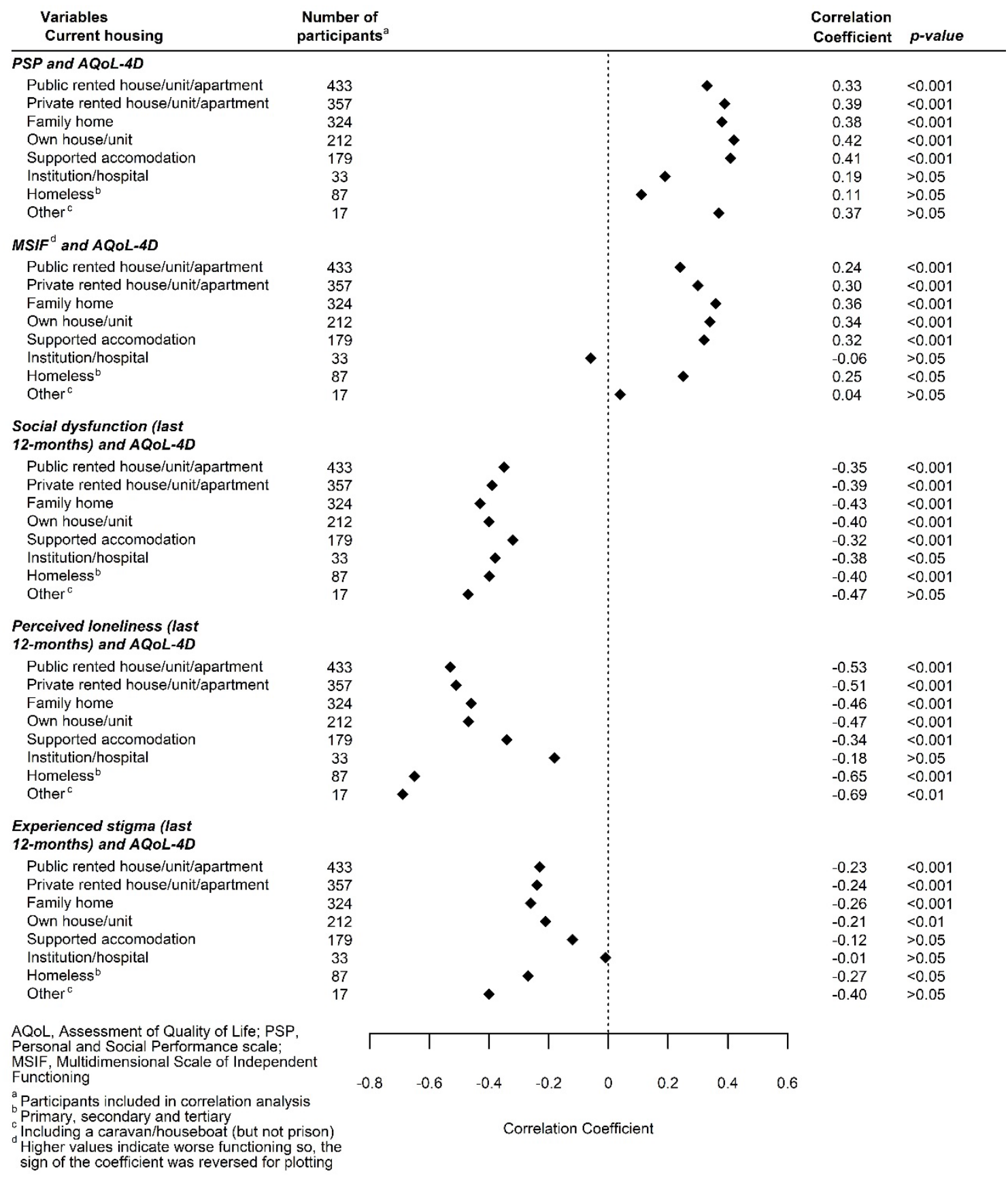

Fig. 1 Correlation coefficients between AQoL-4D utilities and global, independent and social functioning and social experiences in the last 12-months stratified by current housing 
When assessed by 12-month service utilization, most correlations were moderate or higher, except for experienced stigma which were small other than for the ED group, for which they were moderate. Correlations with perceived loneliness were the largest for all groups, except for the GPMH group, for which it was the third largest (Fig. 2). The greatest variation was observed for social dysfunction, followed by global functioning, both ranging from a small correlation ( $r=-0.17, r=0.18$ respectively) for the "Non-specified services" group to the second and fourth largest of all correlations ( $r=-0.68, r=0.55$ respectively) for the ED group. The smallest negligible correlation $(r=-0.08)$ was observed for experienced stigma for the "Non-specified services" group. Associations for the ED group were the strongest across all variables except for independent functioning for which it was second largest $(r=-0.41)$. The smallest association for the ED group was for experienced stigma which was moderate $(r=-0.30)$. Correlations for the GPMH group ranged from small to large ( $r=-0.19$ to -0.59$)$ with the second strongest associations across all groups for global functioning $(r=0.45)$ and social dysfunction $(r=-0.59)$. Associations for MH Inpatient admission and MH Outpatients services were all moderate, with the exception of experienced stigma. Associations for the "Non-specified services" group were the weakest across all groups; all small, except for perceived loneliness, which was moderate $(r=-0.48)$. 


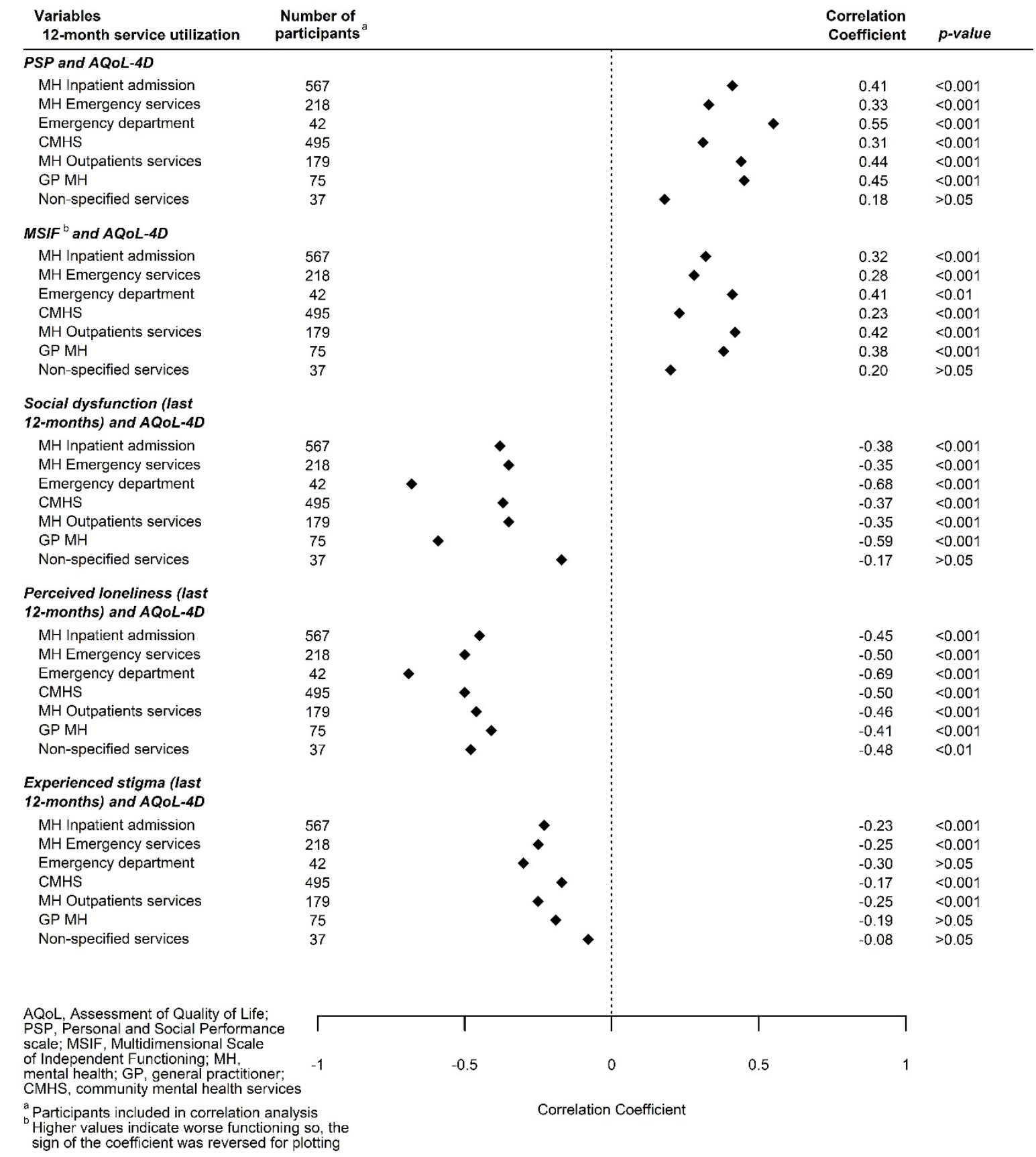

Fig. 2 Correlation coefficients between AQoL-4D utilities and global, independent and social functioning and social experiences in the last 12-months stratified by 12 -months service utilization 
When assessed by course of disorder, correlations for global and independent functioning were particularly striking (Fig. 3). While correlations with global functioning were all moderate and showed little variation, there was a linear trend across the categories for independent functioning, ranging from small for continuous illness with deterioration to moderate for single episode good recovery. As for the entire sample, correlations with perceived loneliness were the largest for each course of disorder sub-group ( $r=-0.40$ to -0.52$)$. There was no group that consistently gave rise to the strongest associations, while continuous illness with deterioration had the lowest or second lowest correlations for each assessment. Correlations for continuous illness without and with deterioration were mostly small $(r=-0.15$ to -0.27$)$. Exceptions were for perceived loneliness where correlations were large for continuous illness without deterioration $(r=-0.52)$ and moderate for continuous illness with deterioration $(r=-0.40)$, and for global functioning for both groups where correlations were moderate ( $r=0.34, r=0.31$ respectively). 


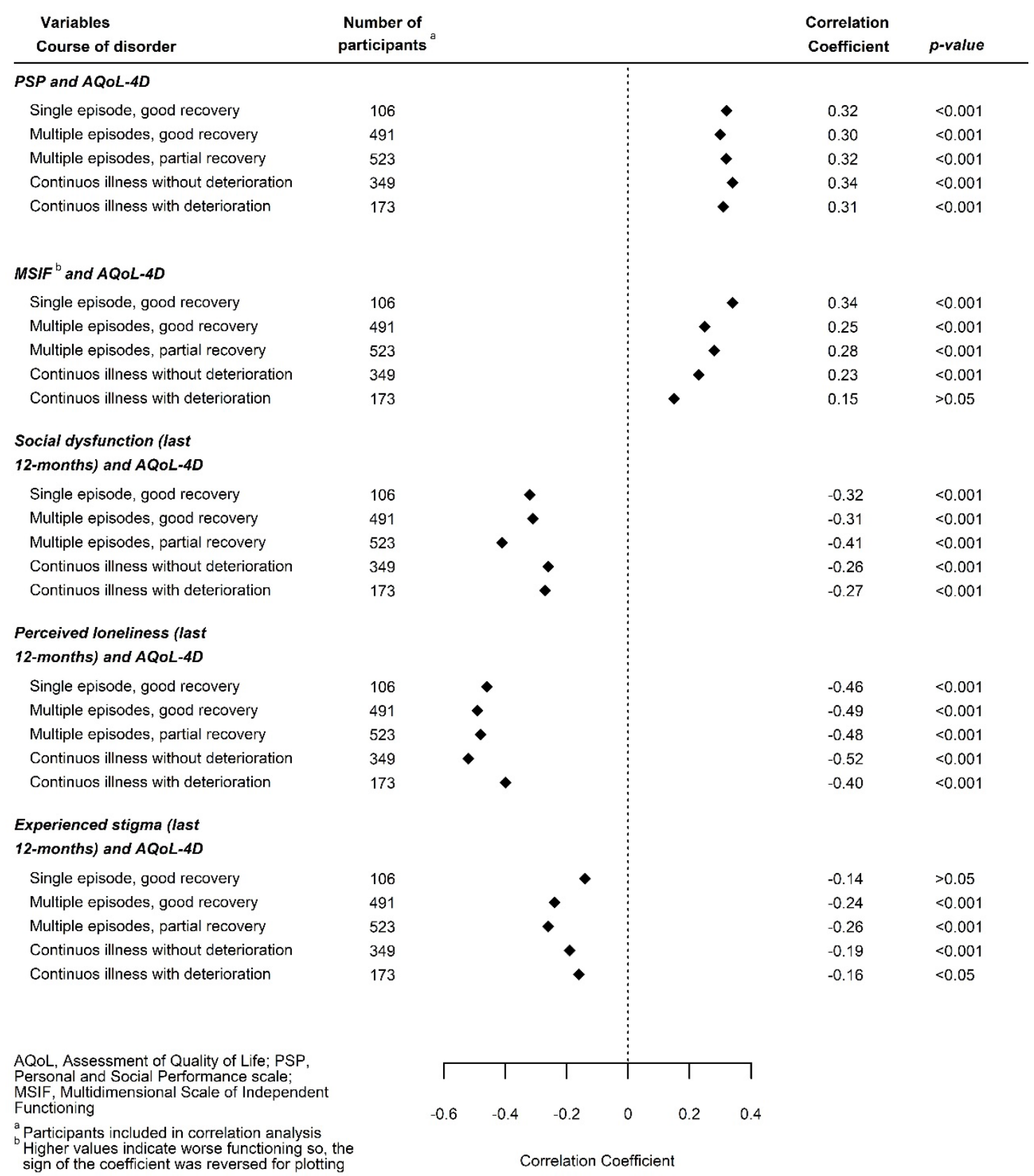

Fig. 3 Correlation coefficients between AQoL-4D utilities and global, independent and social functioning and social experiences in the last 12-months stratified by course of disorder 


\section{Different aspects of functioning as predictors of HRQoL}

Global, independent and social functioning were modelled as individual predictors of AQoL-4D utilities, each aspect of functioning having a statistically significant association and achieving the minimally important difference (Table 2). The highest independent effect was observed for global functioning (Adj R-squared=0.16) then social dysfunction (Adj R-squared=0.15). When jointly considered, independent functioning became non-significant, and the variance explained became $23 \%$ (Table 2).

Table 2 Univariate and multivariate associations between HRQoL and global, independent and social functioning $(\mathrm{n}=1,613)$

\begin{tabular}{|c|c|c|c|c|c|}
\hline Variable & Constant & Coefficient & $95 \% \mathrm{CI}$ & $p$-value & $\overline{\text { Adj. } R^{2}}$ \\
\hline \multicolumn{6}{|l|}{ Univariate analyses } \\
\hline \multicolumn{6}{|l|}{ Global functioning } \\
\hline cons & 0.07 & & $(0.02,0.12)$ & 0.006 & 0.16 \\
\hline PSP & & 0.01 & $(0.01,0.01)$ & 0.000 & \\
\hline PSP (10pts. Interval) & & 0.08 & & & \\
\hline \multicolumn{6}{|c|}{ MSIF Overall global independent functioning } \\
\hline Essentially normal functioning & 0.73 & & $(0.67,0.79)$ & 0.000 & 0.11 \\
\hline Very mild disability & & -0.13 & $(-0.20,-0.07)$ & 0.000 & \\
\hline Somewhat disabled & & -0.20 & $(-0.26,-0.14)$ & 0.000 & \\
\hline Moderately disabled & & -0.27 & $(-0.33,-0.21)$ & 0.000 & \\
\hline Significantly disabled & & -0.31 & $(-0.38,-0.25)$ & 0.000 & \\
\hline Extremely disabled & & -0.45 & $(-0.53,-0.37)$ & 0.000 & \\
\hline Totally disabled & & -0.39 & $(-0.52,-0.25)$ & 0.000 & \\
\hline \multicolumn{6}{|l|}{ Social functioning $(\mathrm{n}=1,612)$} \\
\hline No dysfunction & 0.64 & & $(0.61,0.66)$ & 0.000 & 0.15 \\
\hline Obvious dysfunction & & -0.17 & $(-0.20,-0.14)$ & 0.000 & \\
\hline Severe dysfunction & & -0.29 & $(-0.33,-0.26)$ & 0.000 & \\
\hline
\end{tabular}




\begin{tabular}{|c|c|c|c|c|c|}
\hline Variable & Constant & Coefficient & $95 \% \mathrm{CI}$ & $p$-value & Adj. $R^{2}$ \\
\hline \multicolumn{6}{|c|}{ Multivariate analysis } \\
\hline cons & 0.29 & & $(0.17,0.42)$ & 0.000 & 0.23 \\
\hline \multicolumn{6}{|l|}{ Global functioning } \\
\hline PSP & & 0.006 & $(0.00,0.01)$ & 0.000 & \\
\hline PSP (10pts. Interval) & & 0.06 & & & \\
\hline \multicolumn{6}{|l|}{ Independent functioning } \\
\hline Very mild disability & & -0.04 & $(-0.10,0.02)$ & 0.167 & \\
\hline Somewhat disabled & & -0.04 & $(-0.10,0.03)$ & 0.256 & \\
\hline Moderately disabled & & -0.03 & $(-0.10,0.04)$ & 0.473 & \\
\hline Significantly disabled & & 0.00 & $(-0.08,0.08)$ & 0.924 & \\
\hline Extremely disabled & & -0.07 & $(-0.17,0.03)$ & 0.197 & \\
\hline Totally disabled & & 0.01 & $(-0.14,0.16)$ & 0.864 & \\
\hline \multicolumn{6}{|l|}{ Social functioning } \\
\hline Obvious dysfunction & & -0.13 & $(-0.16,-0.10)$ & 0.000 & \\
\hline Severe dysfunction & & -0.22 & $(-0.25,-0.18)$ & 0.000 & \\
\hline
\end{tabular}

PSP, Personal and Social Performance 


\section{Discussion}

As a preliminary stage towards development of a comprehensive causal model for HRQL in people with psychotic disorders and examining the feasibility of modelling HRQoL using the AQoL-4D, we have analyzed relationships between HRQoL assessed using the AQoL-4D with functioning (global, independent, social) and social experiences (loneliness, experienced stigma) and examined whether those relationships were affected by current housing and clinical status. We also identified which aspects of functioning jointly predicted the HRQoL of study participants.

\section{The relationship between HRQoL and functioning and social experiences}

The associations between HRQoL and global functioning were as hypothesized, and consistent with findings from a recent systematic review (Nevarez-Flores et al. 2019). Likewise, the associations between all aspects of functioning and HRQoL provide further evidence of a robust relationship between these outcomes. However, the predominantly higher absolute value of correlations for global functioning assessed with the PSP than for independent functioning assessed with the MSIF, and a markedly different pattern of correlations for independent functioning with course of disorder, shows that functioning instruments can influence correlations as well as QoL instruments (Nevarez-Flores et al. 2019). Further, these results suggest that specific aspects of functioning may have different impacts on QoL.

The strongest association, between HRQoL and perceived loneliness, indicates that contact with others, or the lack thereof, is an important factor in the life of those living with psychosis. This finding is consistent with previous literature in which loneliness and social isolation have been identified as common difficulties in people with a psychotic disorder (Stain et al. 2012). In contrast, experienced stigma was found to be weakly associated with HRQoL, and all other variables. These weak associations may, in part, be explained by the fact that experienced stigma was assessed as a simple binary variable. However, experienced stigma is also an objective event (Gray 2002) and thus may not contribute as strongly to an individual's perception of QoL as does perceived stigma, a subjective sensation which has been found to be related to QoL (Holubova et al. 2016) and HRQoL (Tang and Wu 2012). Overall, these findings highlight the ability of the AQoL-4D to differentiate between the effects of different aspects of functioning and social experiences on HRQoL. Our findings also arguably obviate the recent statement that "the QALY has not been specifically designed to pick up changes in loneliness (McDaid et al. 2017), and highlights the importance of the sensitivity of the utility instrument employed. 
The hypothesis that the social relationships dimension of the AQoL-4D would be the most strongly related with global functioning, as found for other QoL instruments (Nevarez-Flores et al. 2019), was not confirmed. Rather, the AQoL-4D's independent living dimension had the strongest correlation with global and independent functioning. We thus consider that a HRQoL measure that includes an independent living domain should better capture functioning than those that do not. On the other hand, and consistent with previous research with the AQoL-4D, the social relationships dimension was more strongly related with HRQoL (Herrman et al. 2002), as well as with the social variables, particularly loneliness. Given the differences in associations between dimensions of the AQoL-4D and different aspects of functioning and social experiences, performance of life roles and social relationships appear to contribute separately to HRQoL, and in turn each appear likely targets for strategies aimed at improving HRQoL in people with psychotic disorders.

\section{The impact of housing and clinical status}

Our hypothesis that correlations between HRQoL and functioning and social experiences would differ by housing status was observed for all variables except social functioning. For global and independent functioning people with more stable housing (i.e., living in public or private rented house/unit/apartment, family or own house/unit and supported accommodation) usually had higher correlations with HRQoL than for those living in an institution/hospital or marginal housing. These findings are consistent with higher correlations between functioning and QoL for outpatients than inpatients in (Hosseini and Yousefi 2011; Hunter and Barry 2012; Kusel et al. 2007 ; Ritsner et al. 2005). For social dysfunction and loneliness, the strongest (negative) correlations with HRQoL were observed for the marginal housing group. This finding arguably reflects the importance of human relationships to HRQoL; marginalised people with psychosis having the lowest mean HRQoL across the housing categories (Online Resource Table 3). Further, loneliness and social isolation is an ongoing concern within this group (Harvey et al. 2002).

The results of this study also provide support towards a distinction between the number and quality of relationships and the capacity to engage in those relationships and socialize more generally, as reflected in the institution/hospital category results. This group was the only housing category for which the correlation for perceived loneliness was smaller than for social functioning, and the only category for which the correlation for perceived loneliness was small. Given the low HRQoL in this group (Online Resource Table 3), the low correlation between 
perceived loneliness and HRQoL may be because people in institution/hospitals do not feel lonely, since they tend to maintain relationships with other residents, patients and staff (Breier and Strauss 1984).

When correlations were assessed by 12-month service utilization, the highest correlations were found for those attending ED in the last 12-months, which indicates greater concordance between their HRQoL and functioning and social experiences. People using ED services had the best HRQoL on average (Online Resource Table 3). We postulate this group may consider the ED as a more approachable (Downey et al. 2009) or convenient service, particularly after-hours (Morphet et al. 2012). It may be these individuals are also earlier in the course of their illness, since the ED is the anticipated health service of choice when a person is first in crisis (Morphet et al. 2012).

Conversely, course of disorder made no difference to the assessment of the correlations between HRQoL and functioning assessed with the PSP, but did for the MSIF, reflecting the differences between these two instruments. We consider that the observed gradient in correlations for the MSIF, given its focus on work, is due to adaptation to the effects of unemployment over time and/or a floor effect on QoL impacts; longer duration of illness being assumed in people with continuous illness. Thus, clinical status is an important consideration in the assessment of the relationship between HRQoL, functioning and social experiences.

\section{Aspects of functioning that jointly predict the HRQoL of people with psychotic disorders}

Our hypothesis that global functioning would be the strongest predictor of HRQoL among the functioning variables was observed. Further, the importance of social aspects of functioning for people with psychotic disorders was highlighted when social functioning was found to jointly predict HRQoL with global functioning. Regarding independent functioning, we postulate that its effect was attenuated by global functioning given the strong correlation between these variables. On the basis of our findings, global and social functioning only need be considered when assessing predictors of HRQoL in people living with a psychotic disorder, at least when HRQoL is assessed using the AQoL-4D.

\section{Strengths and Limitations}

Given the purpose of our study is to inform development of a model of HRQoL, the size of the sample for which we have data is a strength, enabling us to consider multiple variables and identify potential confounding as indicated for housing and clinical status. The lack of significance for some variables indicates that they need not be considered in 
future models, while the significance and strength of other correlations, both moderate and small, supports their future inclusion. Finally, as the only strong correlation is between global functioning and independent functioning, these findings support HRQoL as a distinct construct. Study limitations arise from the cross-sectional design of the 2010 Australian National Survey of Psychosis, which prevents us establishing causation between different aspects of functioning and HRQoL. Further, given concerns about respondent burden, some variables such as social functioning, perception of loneliness and experienced stigma were assessed with a single question instead of multi-item instruments. Ultimately, as this was a preliminary (exploratory) analysis, we predominantly assessed simple correlations to establish the bona fides for future analysis. However, variables were required to achieve both significance at $p<0.05$ and the minimally important difference for the AQoL-4D (0.06).

In conclusion, our study provides further evidence of the association between HRQoL and functioning, and the sensitivity of the AQoL-4D in people with psychotic disorders. The strong relationship between the independent living dimension of the AQoL-4D with global and independent functioning, underlines the effect of the performance of life roles on the QoL of people living with psychosis. However, the social dimension was most strongly related to social functioning and HRQoL. Moreover, global and social functioning were found to jointly predict the HRQoL of this population and perception of loneliness had the strongest relationship with HRQoL except for people situated in an institution/hospital, highlighting the importance of real and perceived socializing to HRQoL. Further, the consistent relationship between social functioning and HRQoL across all groups suggests that for people living with psychotic disorders the capacity to socialize and positively engage in social relationships is key to the HRQLL of this population. Thus, global and social functioning as well as perception of loneliness, housing, 12-month service utilization and course of disorder should be included in causal models for HRQoL of people living with psychotic disorders, and this is feasible when HRQoL is assessed with the AQoL-4D. 


\section{References}

Aas, I. M. (2010). Global Assessment of Functioning (GAF): Properties and frontier of current knowledge. Annals of General Psychiatry, 9(1), 20.

American Psychiatric Association (2013). Diagnostic and Statistical Manual of Mental Disorders, Fifth Edition. Arlington, VA: American Psychiatric Association.

AQoL (2014). AQoL Assessment of Quality of Life. www.aqol.com.au. Accessed 2016.

Breier, A., \& Strauss, J. S. (1984). The role of social relationships in the recovery from psychotic disorders. The American Journal of Psychiatry, 141, 949-955.

Chrostek, A., Grygiel, P., Anczewska, M., Wciórka, J., \& Świtaj, P. (2016). The intensity and correlates of the feelings of loneliness in people with psychosis. Comprehensive Psychiatry, 70, 190-199, doi:10.1016/j.comppsych.2016.07.015.

Cohen, J. (1988). Statistical power analysis for the behavioral sciences (2nd ed.). Hillsdale, New Jersey: L. Erlbaum Associates.

Downey, L. V. A., Zun, L. S., \& Jones Gonzales, S. (2009). Utilization of emergency department by psychiatric patients. Primary Psychiatry, 16(4), 60.

Fayers, P. M., \& Machin, D. (2016). Quality of life: The assessment, analysis and reporting of patient-reported outcomes (3rd ed.). Chichester Sussex: Wiley Blackwell.

Gray, A. J. (2002). Stigma in psychiatry. Journal of the Royal Society of Medicine, 95(2), 72-76.

Harvey, C., Evert, H., Herrman, H., Pinzone, T., \& Gureje, O. (2002). Disability, homelessness and social relationships among people living with psychosis in Australia. National Survey of Mental Health and Wellbeing Bulletin (Vol. 5, pp. 92). Canberra Australia: Commonwealth Department of Health and Ageing.

Harvey, C., Killackey, E., Groves, A., \& Herrman, H. (2012). A place to live: Housing needs for people with psychotic disorders identified in the second Australian national survey of psychosis. Australian and New Zealand Journal of Psychiatry, 46(9), 840-850.

Hawthorne, G., \& Osborne, R. (2005). Population norms and meaningful differences for the Assessment of Quality of Life (AQoL) measure. Australian and New Zealand Journal of Public Health, 29(2), 136-142, doi:10.1111/j.1467-842X.2005.tb00063.x. 
Hawthorne, G., Richardson, J., \& Osborne, R. (1999). The Assessment of Quality of Life (AQoL) instrument: A psychometric measure of health-related quality of life. Quality of Life Research, 8(3), 209-224, doi:10.1023/a:1008815005736.

Herrman, H., Hawthorne, G., \& Thomas, R. (2002). Quality of life assessment in people living with psychosis. Social Psychiatry and Psychiatric Epidemiology, 37(11), 510-518, doi:10.1007/s00127-002-0587-y.

Holubova, M., Prasko, J., Latalova, K., Ociskova, M., Grambal, A., Kamaradova, D., et al. (2016). Are self-stigma, quality of life, and clinical data interrelated in schizophrenia spectrum patients? A cross-sectional outpatient study. Patient Preference and Adherence, 10, 265-274.

Hosseini, S. H., \& Yousefi, M. K. (2011). Quality of life and GAF in schizophrenia correlation between quality of life and global functioning in schizophrenia. Iranian Journal of Psychiatry and Behavioral Sciences, 5(2), 120125.

Hunter, R., \& Barry, S. (2012). Negative symptoms and psychosocial functioning in schizophrenia: Neglected but important targets for treatment. European Psychiatry, 27(6), 432-436.

Jaeger, J., Berns, S. M., \& Czobor, P. (2003). The multidimensional scale of independent functioning: A new instrument for measuring functional disability in psychiatric populations. Schizophrenia Bulletin, 29(1), 153168.

Kusel, Y., Laugharne, R., Perrington, S., McKendrick, J., Stephenson, D., Stockton-Henderson, J., et al. (2007). Measurement of quality of life in schizophrenia: A comparison of two scales. Social Psychiatry and Psychiatric Epidemiology, 42(10), 819-823.

Lin, C.-Y., Chang, C.-C., Wu, T.-H., \& Wang, J.-D. (2016). Dynamic changes of self-stigma, quality of life, somatic complaints, and depression among people with schizophrenia: A pilot study applying kernel smoothers. Stigma and Health, 1(1), 29-43, doi:10.1037/sah0000014.

McDaid, D., Bauer, A., \& Park, A.-L. (2017). Making the economic case for investing in actions to prevent and/or tackle loneliness: A systematic review. London: Personal Social Services Research Unit London School of Economics and Political Science.

Morgan, V. A., McGrath, J. J., Jablensky, A., Badcock, J. C., Waterreus, A., Bush, R., et al. (2014). Psychosis prevalence and physical, metabolic and cognitive co-morbidity: Data from the second Australian national survey of psychosis. Psychological Medicine, 44(10), 2163-2176, doi:10.1017/s0033291713002973. 
Morgan, V. A., Waterreus, A., Jablensky, A., Mackinnon, A., McGrath, J. J., Carr, V., et al. (2012). People living with psychotic illness in 2010: The second Australian national survey of psychosis. Australian and New Zealand Journal of Psychiatry, 46(8), 735-752, doi:10.1177/0004867412449877.

Morosini, P. L., Magliano, L., Brambilla, L., Ugolini, S., \& Pioli, R. (2000). Development, reliability and acceptability of a new version of the DSM-IV Social and Occupational Functioning Assessment Scale (SOFAS) to assess routine social functioning. Acta Psychiatrica Scandinavica, 101(4), 323-329.

Morphet, J., Innes, K., Munro, I., O’Brien, A., Gaskin, C. J., Reed, F., et al. (2012). Managing people with mental health presentations in emergency departments: A service exploration of the issues surrounding responsiveness from a mental health care consumer and carer perspective. Australasian Emergency Nursing Journal, 15(3), 148-155, doi:10.1016/j.aenj.2012.05.003.

Neil, A. L., Carr, V. J., Mackinnon, A., Foley, D. L., \& Morgan, V. A. (2018). Health-related quality of life in people living with psychotic illness and factors associated with its variation. Value in Health, 21(8), 1002-1009, doi:10.1016/j.jval.2018.02.012.

Nevarez-Flores, A. G., Sanderson, K., Breslin, M., Carr, V. J., Morgan, V. A., \& Neil, A. L. (2019). Systematic review of global functioning and quality of life in people with psychotic disorders. Epidemiology and Psychiatric Sciences, 28(1), 31-44, doi:10.1017/s2045796018000549.

Petho, B., \& Ban, T. A. (1988). DCR Budapest-Nashville in the diagnosis and classification of functional psychoses. Psychopathology, 21(4-5), 149-240.

Ritsner, M., Kurs, R., Ratner, Y., \& Gibel, A. (2005). Condensed version of the Quality of Life Scale for schizophrenia for use in outcome studies. Psychiatry Research, 135(1), 65-75.

Stain, H. J., Galletly, C. A., Clark, S., Wilson, J., Killen, E. A., Anthes, L., et al. (2012). Understanding the social costs of psychosis: The experience of adults affected by psychosis identified within the second Australian national survey of psychosis. Australian and New Zealand Journal of Psychiatry, 46(9), 879-889, doi:10.1177/0004867412449060.

Tang, I. C., \& Wu, H.-C. (2012). Quality of Life and Self-Stigma in Individuals with Schizophrenia. Psychiatric Quarterly, 83(4), 497-507, doi:10.1007/s11126-012-9218-2.

World Health Organization (1992). The ICD-10 Classification of Mental and Behavioural Disorders: Clinical Description and Diagnostic Guidelines. Geneva: WHO. 
World Health Organization (1997). Programme on mental health: WHOQOL measuring quality of life.. Geneva: WHO. 
Table 1 Health-related quality of life, functioning and social experiences of the sample $(n=1,642)$

\begin{tabular}{|c|c|c|}
\hline Variable & & \\
\hline AQoL-4D utilities ${ }^{\mathrm{a}}$, Mean (SD) & 0.50 & $(0.28)$ \\
\hline \multicolumn{3}{|l|}{ AQoL-4D dimension scores, Mean (SD) } \\
\hline Independent living $(\mathrm{n}=1,623)$ & 0.87 & $(0.19)$ \\
\hline Social relationships $(\mathrm{n}=1,615)$ & 0.65 & $(0.30)$ \\
\hline Physical senses $(n=1,626)$ & 0.94 & $(0.10)$ \\
\hline Psychological wellbeing $(\mathrm{n}=1,626)$ & 0.84 & $(0.18)$ \\
\hline PSP score, Mean (SD) & 56.22 & $(14.71)$ \\
\hline \multicolumn{3}{|c|}{ MSIF Overall Global Independent Functioning, n (\%) } \\
\hline Essentially normal functioning & 87 & $(5.30)$ \\
\hline Very mild disability & 302 & $(18.39)$ \\
\hline Somewhat disabled & 418 & $(25.46)$ \\
\hline Moderately disabled & 454 & $(27.65)$ \\
\hline Significantly disabled & 280 & $(17.05)$ \\
\hline Extremely disabled & 83 & $(5.05)$ \\
\hline Totally disabled & 18 & $(1.10)$ \\
\hline \multicolumn{3}{|c|}{ Social dysfunction in the last 12-months, $\mathrm{n}(\%)$} \\
\hline No dysfunction & 589 & $(35.87)$ \\
\hline Obvious dysfunction & 711 & $(43.30)$ \\
\hline Severe dysfunction & 341 & $(20.77)$ \\
\hline Uncertain & 1 & $(0.06)$ \\
\hline \multicolumn{3}{|c|}{ Perceived loneliness in the last 12 -months, $\mathrm{n}(\%)$} \\
\hline Plenty of friends, not lonely & 322 & $(19.61)$ \\
\hline Friends, lonely occasionally & 512 & $(31.18)$ \\
\hline Some friends but lonely for company & 397 & $(24.18)$ \\
\hline Socially isolated and lonely & 372 & $(22.66)$ \\
\hline NK & 39 & $(2.38)$ \\
\hline
\end{tabular}




\begin{tabular}{lll}
\hline Variable & & \\
\hline Experienced stigma in the last 12-months, $\mathrm{n}(\%)$ & & \\
No & 1,011 & $(61.57)$ \\
Yes & 625 & $(38.06)$ \\
NK & 6 & $(0.37)$ \\
\hline
\end{tabular}

AQoL-4D, Assessment of Quality of Life 4 Dimensions; MSIF, Multidimensional Scale of Independent Functioning; NK, not known; PSP, Personal and Social Performance; SD, standard deviation.

${ }^{\mathrm{a}} \mathrm{n}=1,613$ 
Table 2 Demographic and clinical characteristics of the sample $(n=1,642)$

\begin{tabular}{|c|c|c|}
\hline Characteristic & & \\
\hline \multicolumn{3}{|l|}{ Gender, n (\%) } \\
\hline Male & 1,008 & $(61.39)$ \\
\hline Female & 634 & $(38.61)$ \\
\hline Age, Mean (SD & 38.24 & $(11.09)$ \\
\hline \multicolumn{3}{|l|}{ Marital status, n (\%) } \\
\hline Single, never married & 1,034 & $(62.97)$ \\
\hline Married or de facto & 272 & $(16.57)$ \\
\hline Separated, divorced or widowed & 336 & $(20.46)$ \\
\hline \multicolumn{3}{|l|}{ Education level, n (\%) } \\
\hline Year 12 & 519 & $(31.61)$ \\
\hline Year 11 & 253 & $(15.41)$ \\
\hline Year 10 & 484 & $(29.48)$ \\
\hline Year 9 or below & 366 & $(22.29)$ \\
\hline Other (NK, NA) & 20 & $(1.22)$ \\
\hline \multicolumn{3}{|l|}{ Employment (last 12-months), n (\%) } \\
\hline Employed & 533 & $(32.46)$ \\
\hline Volunteer/unpaid work & 160 & $(9.74)$ \\
\hline Student & 58 & $(3.53)$ \\
\hline No formal activity/Unemployed & 891 & $(54.26)$ \\
\hline \multicolumn{3}{|l|}{ Current housing, n (\%) } \\
\hline Public rented house/unit/apartment & 533 & $(32.46)$ \\
\hline Private rented house/unit/apartment & 357 & $(21.74)$ \\
\hline Family home & 324 & $(19.73)$ \\
\hline Own house/unit & 212 & $(12.91)$ \\
\hline Supported accommodation & 179 & $(10.90)$ \\
\hline Institution/hospital & 33 & $(10.90)$ \\
\hline
\end{tabular}




\begin{tabular}{|c|c|c|}
\hline \multicolumn{3}{|l|}{ Characteristic } \\
\hline Homeless $^{\mathrm{a}}$ & 87 & $(5.30)$ \\
\hline Other (but not prison) ${ }^{b}$ & 17 & $(1.04)$ \\
\hline \multicolumn{3}{|l|}{ Diagnosis ICD-10, n (\%) } \\
\hline Schizophrenia & 857 & $(52.19)$ \\
\hline Schizoaffective disorder & 293 & $(17.84)$ \\
\hline Bipolar disorder with psychotic features & 319 & $(19.43)$ \\
\hline Depressive psychosis & 81 & $(4.93)$ \\
\hline Delusional disorder & 92 & $(5.60)$ \\
\hline Age of onset, Mean (SD) & 23.58 & $(8.46)$ \\
\hline \multicolumn{3}{|l|}{ 12-month service utilization, n (\%) } \\
\hline MH Inpatient admission & 578 & $(35.20)$ \\
\hline MH Emergency services & 220 & $(13.40)$ \\
\hline Emergency department & 44 & $(2.68)$ \\
\hline Community mental health services & 502 & $(30.57)$ \\
\hline MH Outpatients services & 182 & $(11.08)$ \\
\hline GP MH & 76 & $(4.63)$ \\
\hline Non-specified services & 40 & $(2.44)$ \\
\hline \multicolumn{3}{|l|}{ Course of disorder, $\mathrm{n}(\%)$} \\
\hline Single episode (good recovery) & 106 & $(6.46)$ \\
\hline Multiple episodes (good recovery) & 491 & $(29.90)$ \\
\hline Multiple episodes (partial recovery) & 523 & $(31.85)$ \\
\hline Continuous illness without deterioration & 349 & $(21.25)$ \\
\hline Continuous illness with deterioration & 173 & $(10.54)$ \\
\hline Duration of illness, years, Mean (SD) & 14.66 & $(10.24)$ \\
\hline
\end{tabular}

GP, general practitioner; $\mathrm{MH}$, mental health; NA, not available; NK, not known; SD, standard deviation 
${ }^{a}$ Homeless. Primary: living on the streets, in parks or in deserted buildings; secondary: living in temporary shelters such as refuge, emergency accommodation or sleeping on friend's couch; tertiary: private boarding room

${ }^{\mathrm{b}}$ Including living in a caravan/houseboat 
Table 3 HRQoL measured with the AQoL-4D by stratification categories: a) current housing, b) 12-month service utilization and c) course of disorder

\begin{tabular}{|c|c|c|c|c|c|}
\hline \multirow{2}{*}{\multicolumn{2}{|c|}{ Stratification categories }} & \multicolumn{4}{|c|}{ HRQoL } \\
\hline & & \multirow{2}{*}{$\begin{array}{c}\text { Mean } \\
0.47\end{array}$} & \multirow{2}{*}{$\begin{array}{c}\text { SD } \\
0.29\end{array}$} & \multirow{2}{*}{$\begin{array}{r}\text { Median } \\
0.46\end{array}$} & \multirow{2}{*}{$\begin{array}{c}\text { Variance } \\
0.08\end{array}$} \\
\hline \multirow{8}{*}{ 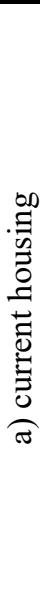 } & Public rented house/unit/apartment & & & & \\
\hline & Private rented house/unit/apartment & 0.51 & 0.27 & 0.52 & 0.07 \\
\hline & Family home & 0.57 & 0.27 & 0.60 & 0.07 \\
\hline & Own house/unit & 0.56 & 0.29 & 0.60 & 0.08 \\
\hline & Supported accommodation & 0.44 & 0.29 & 0.46 & 0.08 \\
\hline & Institution/hospital & 0.31 & 0.22 & 0.26 & 0.05 \\
\hline & Homeless $^{\mathrm{a}}$ & 0.47 & 0.28 & 0.48 & 0.08 \\
\hline & Other (but not prison) ${ }^{b}$ & 0.45 & 0.31 & 0.41 & 0.10 \\
\hline \multirow{7}{*}{ 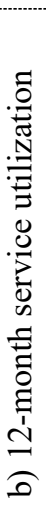 } & MH Inpatient admission & 0.46 & 0.29 & 0.45 & 0.08 \\
\hline & MH Emergency services & 0.47 & 0.28 & 0.48 & 0.08 \\
\hline & Emergency department & 0.60 & 0.30 & 0.64 & 0.09 \\
\hline & Community mental health services & 0.55 & 0.27 & 0.60 & 0.07 \\
\hline & MH Outpatients services & 0.55 & 0.26 & 0.57 & 0.07 \\
\hline & GP MH & 0.46 & 0.30 & 0.48 & 0.09 \\
\hline & Non-specified services & 0.48 & 0.28 & 0.43 & 0.08 \\
\hline \multirow{5}{*}{ 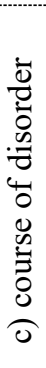 } & Single episode (good recovery) & 0.63 & 0.27 & 0.68 & 0.07 \\
\hline & Multiple episodes (good recovery) & 0.60 & 0.26 & 0.65 & 0.07 \\
\hline & Multiple episodes (partial recovery) & 0.49 & 0.28 & 0.50 & 0.07 \\
\hline & Continuous illness without deterioration & 0.43 & 0.28 & 0.42 & 0.08 \\
\hline & Continuous illness with deterioration & 0.33 & 0.28 & 0.22 & 0.08 \\
\hline
\end{tabular}

AQoL-4D, Assessment of Quality of Life; GP, general practitioner; HRQoL, health-related quality of life; $\mathrm{MH}$, mental health; SD, standard deviation 
${ }^{a}$ Homeless. Primary: living on the streets, in parks or in deserted buildings; secondary: living in temporary shelters such as refuge, emergency accommodation or sleeping on friend's couch; tertiary: private boarding room

${ }^{\mathrm{b}}$ Including living in a caravan/houseboat 